\title{
Highly efficient light emission from stacking faults intersecting nonpolar GalnN quantum wells
}

\author{
H. Jönen, ${ }^{1, a)}$ U. Rossow, ${ }^{1}$ H. Bremers, ${ }^{1}$ L. Hoffmann, ${ }^{1}$ M. Brendel, ${ }^{1}$ A. D. Dräger, ${ }^{1}$ \\ S. Schwaiger, ${ }^{2}$ F. Scholz, ${ }^{2}$ J. Thalmair, ${ }^{3}$ J. Zweck, ${ }^{3}$ and A. Hangleiter ${ }^{1}$ \\ ${ }^{1}$ Institute of Applied Physics, Technische Universität Braunschweig, Mendelssohnstrasse 2, \\ 38106 Braunschweig, Germany \\ ${ }^{2}$ Institute of Optoelectronics, University of Ulm, Albert-Einstein-Allee 45, 89081 Ulm, Germany \\ ${ }^{3}$ Institute of Experimental and Applied Physics, University of Regensburg, 93040 Regensburg, Germany
}

(Received 28 March 2011; accepted 12 June 2011; published online 5 July 2011)

\begin{abstract}
We report on the optical properties of m-plane GaInN/GaN quantum wells (QWs). We found that the emission energy of GaInN QWs grown on m-plane $\mathrm{SiC}$ is significantly lower than on nonpolar bulk GaN, which we attribute to the high density of stacking faults. Temperature and power dependent photoluminescence reveals that the GaInN QWs on $\mathrm{SiC}$ have almost as large internal quantum efficiencies as on bulk GaN despite the much higher defect density. Our results indicate that quantum-wire-like features formed by stacking faults intersecting the quantum wells provide a highly efficient light emission completely dominating the optical properties of the structures. (C) 2011 American Institute of Physics. [doi:10.1063/1.3607301]
\end{abstract}

In the past few years, GaN-based light emitting devices grown on non-polar planes have continuously attracted increasing attention due to their promising optical properties. While conventional structures grown on the polar c-plane suffer from the quantum-confined Stark effect (QCSE), ${ }^{1}$ $\mathrm{GaN}$ layers grown on non-polar surfaces are free from polarization fields in growth direction. ${ }^{2}$ The increased transition probability may result in an improved device efficiency leading to increased light output powers and/or reduced threshold current densities. ${ }^{3}$ Indeed, the first GaInN based laser diodes with an emission wavelength at $500 \mathrm{~nm}$ have been shown by Okamoto et al. on m-plane GaN substrates. ${ }^{4}$ However, up to now, non-polar GaN substrates are still barely available, small in size, and also very expensive. As an alternative, several groups have reported heteroepitaxial growth of non-polar GaN layers on foreign substrates $\left(\gamma-\mathrm{LiAlO}_{2}, \mathrm{SiC}\right.$, r-plane sapphire). ${ }^{5-7}$ However, most of these structures were affected by high densities of threading dislocations (TDs) and basal plane stacking faults (BSFs), which are terminated by either prismatic stacking faults (PSFs) or partial dislocations. ${ }^{8}$ While TDs are known to act as nonradiative recombination centers, BSFs are optically active since they can be considered as cubic (zincblende) $A B C$ phases in the wurtzite $A B A B$ stacking sequence. Such a structure forms a type-II heterojunction which may capture electrons and holes resulting in optical transitions below the wurtzite GaN bandgap energy. ${ }^{9-11}$ More recently, a BSF related emission from aplane GaN/AlGaN quantum well (QW) structures was reported, suggesting the formation of quantum-wire-like states in the regions where BSFs intersect the QWs. ${ }^{12,13}$ In this paper, we investigate the optical properties of m-plane GaInN/GaN QW structures grown on silicon carbide $(\mathrm{SiC})$. In particular, we show that stacking faults intersecting the QWs dominate the optical properties of these structures.

Our samples were grown on m-plane $6 \mathrm{H}-\mathrm{SiC}$, m-plane bulk $\mathrm{GaN}$ substrates, and a-plane GaN templates (grown by

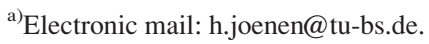

hydride vapor phase epitaxy) in a low pressure metalorganic vapor phase epitaxy system with a horizontal reactor (Aixtron AIX 200RF). The precursors used were trimethylgallium, triethylgallium, trimethylaluminum, trimethylindium, and ammonia. On SiC, a $100 \mathrm{~nm}$ high temperature AlN layer was followed by a $1.1 \mu \mathrm{m}$ thick n-type doped GaN buffer layer grown at $1180^{\circ} \mathrm{C}$ with a low V/III ratio of 700 and a total reactor pressure of 50 mbar. For the active region, the carrier gas was switched from $\mathrm{H}_{2}$ to $\mathrm{N}_{2}$. The single or multiple GaInN QWs were then grown at temperatures between $700^{\circ} \mathrm{C}$ and $800^{\circ} \mathrm{C}$. After growth, the samples were characterized by $\mathrm{x}$-ray diffraction (XRD), transmission electron microscopy (TEM), conventional photoluminescence (PL) at room and low temperatures $(15 \mathrm{~K})$, and high excitation PL (excitation power density several $\mathrm{MW} / \mathrm{cm}^{2}$ ). The internal quantum efficiency (IQE) was determined from temperature and power dependent PL measurements. ${ }^{14}$

The structural quality of the samples was studied by XRD measurements. The procedure to deduce lattice constants, strain states, and finally the indium concentration in the QWs is described in Ref. 15. The latter was found to be between $10 \%$ and $37 \%$ depending on the respective growth temperature (see also Ref. 16). Strain state analyses revealed that the use of thin QWs $\left(\mathrm{d}_{Q W}=(1.5 \pm 0.2) \mathrm{nm}\right)$ results in a small degree of relaxation well below $10 \%$, which allows us to treat the structures as fully strained in the further discussion. In addition, a modified Williamson-Hall analysis of the $\mathrm{x}$-ray rocking curve widths ${ }^{17}$ was used to determine the stacking fault density in our samples. While the BSF density of the structures on bulk GaN was below the detection limit of about $1 \times 10^{4} \mathrm{~cm}^{-1},{ }^{17}$ we found a stacking fault density of about $1 \times 10^{6} \mathrm{~cm}^{-1}$ in the samples on m-plane $\mathrm{SiC}$ in good agreement with TEM measurements. Fig. 1 shows room temperature PL spectra of GaInN/GaN $5 \times \mathrm{QW}$ structures on $\mathrm{SiC}$ grown at different temperatures. With decreasing growth temperature, a decreasing emission energy is observed corresponding to the higher indium incorporation in the QWs. 


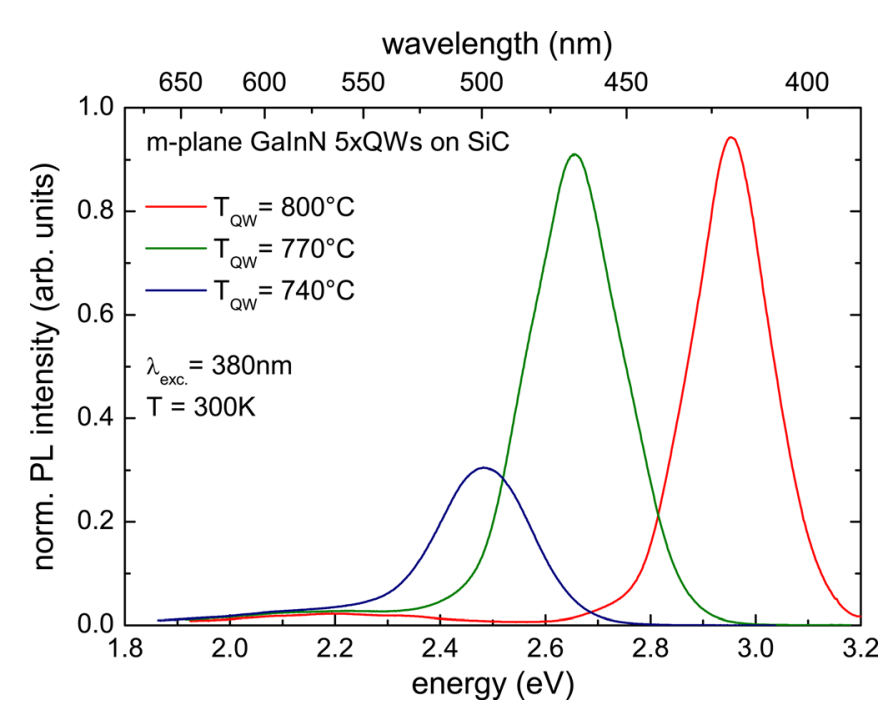

FIG. 1. (Color online) Room temperature PL measurements of m-plane GaInN/GaN $5 \times$ QW structures with different indium concentrations resulting from different growth temperatures.

The optical transition energies from the PL measurements at low temperature are shown in Fig. 2 as a function of the In content determined by XRD. For the simulation shown in the figure (solid line), we used a bandgap energy (of unstrained material) of $3.51 \mathrm{eV}$ for GaN (Ref. 18) and $0.67 \mathrm{eV}$ for InN (Ref. 19) with a bowing parameter of 2.6 $\mathrm{eV}$, which is close to the recently reported value by Moret et $a l .{ }^{20}$ To take the strain induced shift of the bandgap energy into account, we used the relations given by Shan et al. ${ }^{21}$ with a linear interpolation of the elastic constants ${ }^{22}$ and the deformation potentials. $^{23,24}$ The optical transition energies (including exciton binding energies) were then calculated for a QW thickness of $1.5 \mathrm{~nm}$ by numerically solving Schrödinger's equation assuming a band offset ratio of $\Delta E_{C}$ ' $\Delta E_{V}=70: 30$ (Ref. 25) and electron and hole effective masses of $0.2 m_{0}$ and $2.0 m_{0},{ }^{26}$ respectively. The error margin of the calculations is indicated as the shaded area in Fig. 2, given an accuracy of $\pm 0.2 \mathrm{~nm}$ (Ref. 15) in the determination of the QW thickness. Good agreement can be observed between the data points on non-polar bulk GaN and the

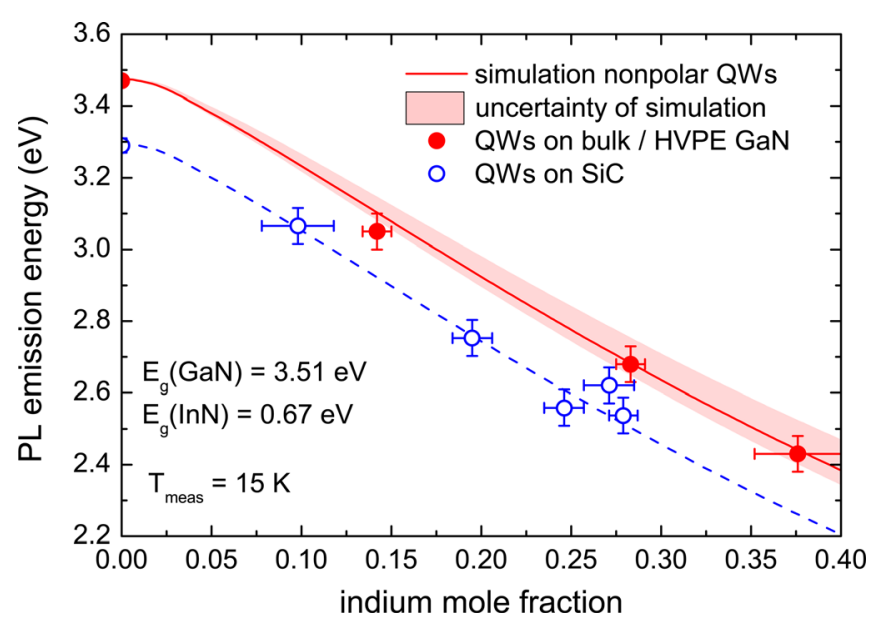

FIG. 2. (Color online) Low temperature PL emission energies of nonpolar GaInN QWs and GaN buffer layers (at $x=0$ ) as a function of In content. An unexpectedly small emission energy is observed for the m-plane GaInN/ $\mathrm{GaN} 5 \times \mathrm{QWs}$ on m-plane SiC substrates. Dashed lines are guide to the eye. calculated transition energies. For the m-plane samples on $\mathrm{SiC}$, however, the apparent bandgap is about $180 \mathrm{meV}$ smaller than expected from the calculations. In addition, the QW structures on m-plane $\mathrm{SiC}$ show an unexpected blueshift of the PL emission energy under high excitation as it is known from the screening of electric fields in a polar QW, whereas the emission energy of the structures on bulk GaN is independent of the excitation power.

Obviously, the emission of the structures on m-plane $\mathrm{SiC}$ relates to the QWs, as the energy scales with incorporated In. On the other hand, the emission energy is lower than expected by about the same energy difference as stacking fault emissions in $\mathrm{GaN}$ are lower in energy than the bandgap. Investigating the PL spectra of the GaN buffer layers at low temperature (not shown), we find the dominating emission at around $3.3 \mathrm{eV}$ (see Fig. 2). In addition, a stacking fault related peak and the near-band-edge emission are observed at around $3.4 \mathrm{eV}$ and $3.49 \mathrm{eV}$, respectively. While the $3.4 \mathrm{eV}$ emission is already clearly associated with BSFs of type $I_{1},{ }^{27,28}$ there is still some discussion about the $3.3 \mathrm{eV}$ emission. However, it is most likely attributed to BSFs of type $I_{2}{ }^{29}$ or prismatic stacking faults, ${ }^{27,30}$ which can arise from folded BSFs with stair-rod dislocations at their intersections. ${ }^{8,30}$ Now, as we mentioned above, there are high densities of stacking faults in our heteroepitaxial samples. Therefore, it is reasonable to assume that not only the GaN emission but also the QW emission is influenced by the stacking faults. This is supported by the fact that the nonpolar structures on bulk GaN with a very low stacking fault density have a higher PL emission energy, which corresponds to the calculated values. Furthermore, the observed blueshift of the PL emission of the heteroepitaxial samples under high excitation may then be a consequence of an electric field in the cubic type-II QWs induced by the spontaneous polarization of the wurtzite matrix in the [0001]direction, which is perpendicular to the stacking faults. ${ }^{11}$

TEM measurements reveal that BSFs are intersecting the quantum wells (see Fig. 3). At the intersection, a rectangular region is formed, where confinement both by the quantum well and by the stacking fault occurs. These regions may, therefore, be regarded as quantum-wire-like oriented along the a-direction. Indeed, the formation of quantum wires by stacking faults intersecting the quantum wells has already been suggested for a-plane GaN/AlGaN QW structures, ${ }^{12,13}$ and most likely, it also applies for our m-plane
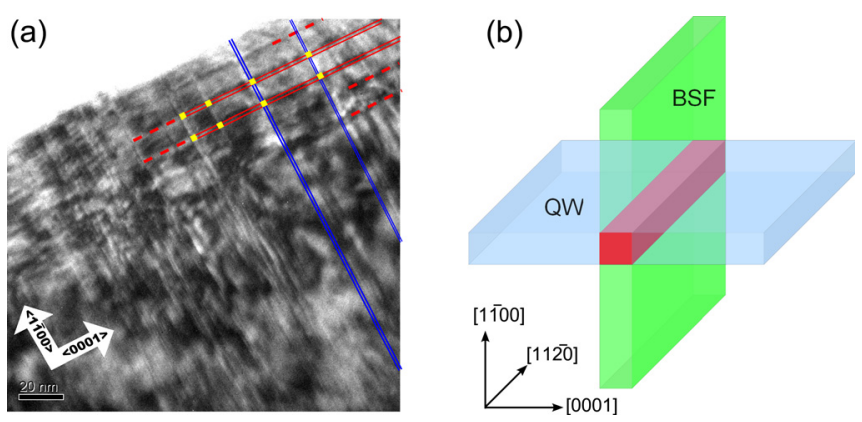

FIG. 3. (Color online) Intersections of stacking faults and QWs. (a) TEM image of a GaInN/GaN $5 \times$ QW. (b) Schematic view of a m-plane QW intersecting a basal plane stacking fault. 


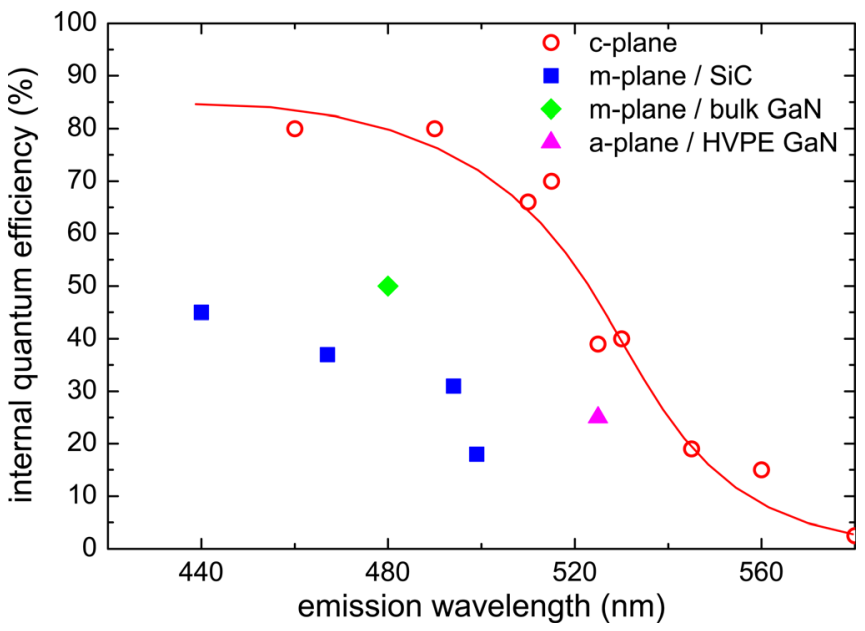

FIG. 4. (Color online) Room temperature internal quantum efficiencies of c-plane and m-plane GaInN/GaN QW structures.

GaInN/GaN QWs. In this context, it should be noted that in all our experiments with the samples on m-plane $\mathrm{SiC}$ (even at highest excitation powers), no other emission at higher energy was observed, which could be attributed to the "real" wurtzite bandgap. This may be explained by a very efficient confinement of electrons and holes within the quantum wire region. On the other hand, such a mechanism should also affect the internal quantum efficiency of these structures.

Fig. 4 shows the internal quantum efficiencies of c-plane and m-plane GaInN/GaN QW structures as a function of the emission wavelength. The structures on m-plane $\mathrm{SiC}$ have reasonably large quantum efficiencies up to $45 \%$ at room temperature, which is almost as high as the values we obtained for QWs on m-plane bulk GaN. At first glance, this is surprising given the large defect density on m-plane $\mathrm{SiC}$ compared to that on the bulk GaN substrates. However, it clearly demonstrates how effectively carriers may be confined in the quantum wire region resulting in strong radiative recombination and a high quantum efficiency. On the other hand, the efficiency of the m-plane structures is still below the values of our optimized c-plane QWs. However, c-plane QWs may greatly benefit from V-shaped pits suppressing nonradiative recombination, ${ }^{31}$ which is likely not the case for m-plane structures.

In conclusion, we have investigated the optical properties of m-plane GaInN/GaN QW structures. Comparing PL transition energies with XRD data, we have found an unexpectedly low effective bandgap energy for samples on $\mathrm{m}$ plane $\mathrm{SiC}$, which we attributed to the stacking faults in our samples. In addition, we observed large internal quantum efficiencies up to $45 \%$ at room temperatures despite the high defect density on the m-plane SiC substrates. We explain this by stacking faults forming quantum-wire-like states in the QW region, which effectively confine carriers and thus dominate the overall optical properties of these structures.

The authors gratefully acknowledge financial support by the DFG in the framework of the Research Group FOR957 Polarization Field Control in Nitride Light Emitters and by the Braunschweig International Graduate School of Metrology (IGSM).

${ }^{1}$ J. S. Im, H. Kollmer, J. Off, A. Sohmer, F. Scholz, and A. Hangleiter, Phys. Rev. B 57, R9435 (1998).

${ }^{2}$ P. Waltereit, O. Brandt, A. Trampert, H. T. Grahn, J. Menniger, M. Ramsteiner, M. Reiche, and K. H. Ploog, Nature 406, 865 (2000).

${ }^{3}$ T. Takeuchi, H. Amano, and I. Akasaki, Jpn. J. Appl. Phys. 39, 413 (2000).

${ }^{4}$ K. Okamoto, J. Kashiwagi, T. Tanaka, and M. Kubota, Appl. Phys. Lett. 94, 071105 (2009).

${ }^{5} \mathrm{~K}$. Horino et al., "Growth of (1100) Oriented GaN on (1100) 6H-SiC by Metalorganic Vapor Phase Epitaxy", International Symposium on Blue Laser and Light Emitting Diodes, Chiba Univ., Japan, Mar. 5-7, 1996, pp. 530-533.

${ }^{6}$ E. S. Hellman, Z. Liliental-Weber, and D. N. E. Buchanan, MRS Internet J. Nitride Semicond. Res. 2, 30 (1997).

${ }^{7}$ M. D. Craven, S. H. Lim, F. Wu, J. S. Speck, and S. P. DenBaars, Appl. Phys. Lett. 81, 469 (2002)

${ }^{8}$ D. N. Zakharov, Z. Liliental-Weber, B. Wagner, Z. J. Reitmeier, E. A. Preble, and R. F. Davis, Phys. Rev. B 71, 235334 (2005).

${ }^{9}$ Y. T. Rebane, Y. G. Shreter, and M. Albrecht, Phys. Status Solidi A 164, 141 (1997).

${ }^{10}$ C. Stampfl and C. G. Van de Walle, Phys. Rev. B 57, R15052 (1998).

${ }^{11}$ Y. J. Sun, O. Brandt, U. Jahn, T. Y. Liu, A. Trampert, S. Cronenberg, S. Dhar, and K. H. Ploog, J. Appl. Phys. 92, 5714 (2002).

${ }^{12}$ T. J. Badcock, P. Dawson, M. J. Kappers, C. McAleese, J. L. Hollander, C. F. Johnston, D. V. S. Rao, A. M. Sanchez, and C. J. Humphreys, Appl. Phys. Lett. 93, 101901 (2008).

${ }^{13}$ P. Corfdir, P. Lefebvre, L. Balet, S. Sonderegger, A. Dussaigne, T. Zhu, D. Martin, J.-D. Ganière, N. Grandjean, and B. Deveaud-Plédran, J. Appl. Phys. 107, 043524 (2010).

${ }^{14}$ A. Hangleiter, D. Fuhrmann, M. Grewe, F. Hitzel, G. Klewer, S. Lahmann, C. Netzel, N. Riedel, and U. Rossow, Phys. Status Solidi A 201, 2808 (2004).

${ }^{15}$ H. Bremers, A. Schwiegel, L. Hoffmann, H. Jönen, U. Rossow, J. Thalmair, J. Zweck, and A. Hangleiter, Phys. Status Solidi B 248, 616 (2011).

${ }^{16}$ H. Jönen, U. Rossow, H. Bremers, L. Hoffmann, M. Brendel, A. D. Dräger, S. Metzner, F. Bertram, J. Christen, S. Schwaiger, F. Scholz, J. Thalmair, J. Zweck, and A. Hangleiter, Phys. Status Solidi B 248, 600 (2011).

${ }^{17}$ M. B. McLaurin, A. Hirai, E. Young, F. Wu, and J. S. Speck, Jpn. J. Appl. Phys. 47, 5429 (2008).

${ }^{18}$ J. F. Muth, J. H. Lee, I. K. Shmagin, R. M. Kolbas, J. H. C. Casey, B. P. Keller, U. K. Mishra, and S. P. DenBaars, Appl. Phys. Lett. 71, 2572 (1997).

${ }^{19}$ A. A. Klochikhin, V. Y. Davydov, V. V. Emtsev, A. V. Sakharov, V. A. Kapitonov, B. A. Andreev, H. Lu, and W. J. Schaff, Phys. Rev. B 71, 195207 (2005).

${ }^{20}$ M. Moret, B. Gil, S. Ruffenach, O. Briot, C. Giesen, M. Heuken, S. Rushworth, T. Leese, and M. Succi, J. Cryst. Growth 311, 2795 (2009).

${ }^{21}$ W. Shan, R. J. Hauenstein, A. J. Fischer, J. J. Song, W. G. Perry, M. D. Bremser, R. F. Davis, and B. Goldenberg, Phys. Rev. B 54, 13460 (1996).

${ }^{22}$ F. Morales, D. Gonzlez, J. Lozano, R. Garca, S. Hauguth-Frank, V. Lebedev, V. Cimalla, and O. Ambacher, Acta Mater. 57, 5681 (2009).

${ }^{23}$ I. Vurgaftman and J. R. Meyer, J. Appl. Phys. 94, 3675 (2003).

${ }^{24}$ B. Gil, M. Moret, O. Briot, S. Ruffenach, C. Giesen, M. Heuken, S. Rushworth, T. Leese, and M. Succi, J. Cryst. Growth 311, 2798 (2009).

${ }^{25}$ T. Das, S. Kabi, and D. Biswas, J. Appl. Phys. 105, 046101 (2009).

${ }^{26}$ J. S. Im, A. Moritz, F. Steuber, V. Härle, F. Scholz, and A. Hangleiter, Appl. Phys. Lett. 70, 631 (1997).

${ }^{27}$ R. Liu, A. Bell, F. A. Ponce, C. Q. Chen, J. W. Yang, and M. A. Khan, Appl. Phys. Lett. 86, 021908 (2005).

${ }^{28}$ J. Mei, S. Srinivasan, R. Liu, F. A. Ponce, Y. Narukawa, and T. Mukai, Appl. Phys. Lett. 88, 141912 (2006)

${ }^{29}$ I. Tischer, M. Feneberg, M. Schirra, H. Yacoub, R. Sauer, K. Thonke, T. Wunderer, F. Scholz, L. Dieterle, E. Müller, and D. Gerthsen, Phys. Rev. B 83, 035314 (2011).

${ }^{30}$ J. Bai, M. Dudley, L. Chen, B. J. Skromme, B. Wagner, R. F. Davis, U. Chowdhury, and R. D. Dupuis, J. Appl. Phys. 97, 116101 (2005).

${ }^{31}$ A. Hangleiter, F. Hitzel, C. Netzel, D. Fuhrmann, U. Rossow, G. Ade, and P. Hinze, Phys. Rev. Lett. 95, 127402 (2005). 